\title{
Neutrophil extracellular traps (Nets) impact upon autoimmune disorders
}

\author{
MICHAE PRZEMYSEAW PRUCHNIAK ${ }^{1,2}$, IWONA KOTUŁA ${ }^{2}$, ANETA MANDA-HANDZLIK ${ }^{1,2}$ \\ ${ }^{1}$ Postgraduate School of Molecular Medicine, Warsaw, Poland \\ ${ }^{2}$ Department of Laboratory Diagnostic and Clinical Immunology of Developmental Age Medical University of Warsaw, Warsaw, Poland
}

\begin{abstract}
Friend or foe? This is often asked question when it comes to neutrophil extracellular traps studies. There is no simple answer to that. At the time of their discovery they were considered to be protectors of our well-being. Excellent pathogen fighting skills were described as purely beneficial. But it was not long before those guardians of immunity reveal their dark side. What seemed to be profitable could also take its toll. They are perfectly constructed, made from nucleic deoxyribonucleic acid ornamented with cytoplasmic and granular proteins, to fight invaders. But this unique build is prone to become considered by our body as a threat. Since there is a thin line which when crossed turns a savior into enemy, it was postulated that Nets can play a significant role in autoimmune disorders pathogenesis and disease exacerbation. Recent years have brought a new insight into autoimmune disorders trying to connect the old knowledge and suspicions with modern discoveries.
\end{abstract}

Key words: rheumatoid arthritis, systemic lupus erythematosus, autoimmunity, neutrophils, neutrophil extracellular traps, small-vessel vasculitis.

(Centr Eur J Immunol 2015; 40 (2): 217-224)

\section{Introduction}

The polymorphonuclear leukocytes (PMNs), or neutrophils have for a long time been considered as the primary soldiers of the innate immune system. As the body main infantry, they are the most abundant fraction of white blood cells in mammals. They possess wide repertoire of abilities used in the war against invading pathogens. Moreover, many studies has shown the connection of these cells to inflammation and host defence. Especially the mechanisms of phagocytosis, nicotinamide adenine dinucleotide phosphate (NADPH) - dependent oxidative burst, and degranulation of antimicrobial proteins have been elucidated in great detail $[1,2]$. Recent years have brought new discoveries to the cells we thought we know everything about. Presence of chromatin fibers ornamented with variety of intracellular proteins in the extracellular matrix after neutrophils activation and death were observed lately. To some extent, this knowledge is not new, these structures have been noticed and described in the past two decades. But it was not until 2004, when Brinkmann team gave a meaningful explanation to this phenomenon [3-5]. Since then, the new trend in immunology has begun. Now, neutrophil extracellular traps or Nets as they are called, are widely examined by the scientists. This unique feature of neutrophil serves as a powerful weapon of defence but also has a darker side, for it can be used against the body own structures enforcing autoimmune disorders. In this review, we shall focus on the controversial aspects of Nets release and the gaps in our knowledge, especially stressing the role of reactive oxygen species as true mediators of Nets generation.

\section{Neutrophil extracellular traps (Nets)}

Extracellular traps (Ets) can be created not only by neutrophils, but also can derived from monocytes, eosinophils and mast cells. Extracellular traps act as primary, not specified immune response to pathogens which body bring on board in the early stages of infection. Beside forming Nets neutrophils have more sophisticated ways of dealing with invaders, like respiratory burst and release of microbicidal proteins from inner granules. Moreover the mechanism of phagocytosis, which is an efficient way of not only microbes removal but also clearing the body from cellular debris, is extensively used. So, why extracellular traps are widely used? It is because they are exclusive, they are powerful and can trap and nullify large bio-structures like fungi or protozoa which cannot be internalized by phagocytes. They also are created in the first hour of microbial contamination, therefore creating the first wall of defence [2].

Correspondence: Mr. Michał Przemysław Pruchniak, Department of Laboratory Diagnostic and Clinical Immunology of Developmental Age Medical University of Warsaw, Warsaw, Poland, e-mail: michal_pruchniak@yahoo.com 
Table 1. Common Netosis inducers

\begin{tabular}{|c|c|}
\hline $\begin{array}{l}\text { Bacteria, fungi, viruses, } \\
\text { protozoa }\end{array}$ & Organic factors \\
\hline $\begin{array}{l}\text { Staphylococcus aureus } \\
\text { Streptococci pyogenes, S. } \\
\text { pneumonia } \\
\text { Aspergillus fumigatus, A. } \\
\text { nidulans } \\
\text { Candidia glabrata } \\
\text { Leishmania } \\
\text { Toxoplasma gondii } \\
\text { Influenza } \\
\text { Human immunodeficiency } \\
\text { virus (HIV) }\end{array}$ & $\begin{array}{l}\text { Lipopolysaccharide (LPS) } \\
\text { Lipophosphoglycan (LPG) } \\
\text { Immune complexes } \\
\text { Activated platelets } \\
\text { Phorbol myristate acetate (PMA) } \\
\text { N-Formylmethionyl-leucyl- } \\
\text { phenylalanine (fMLP) } \\
\text { Platelet activating factor (PAF) } \\
\text { Alumn } \\
\text { Calcium ionophore (Ionomycyn) } \\
\text { Glucose oxidase }\end{array}$ \\
\hline Cytokines & Inorganic factors \\
\hline $\begin{array}{l}\text { Interleukin-8 } \\
\text { Tumor necrosis factor } \\
\text { (TNF- } \alpha \text { ) } \\
\text { MIP-2 (CXCL2) }\end{array}$ & $\begin{array}{l}\text { Calcium ions } \\
\text { Nitric oxide (NO) } \\
\text { Hydrogen peroxide }\left(\mathrm{H}_{2} \mathrm{O}_{2}\right)\end{array}$ \\
\hline
\end{tabular}

The mechanism in which traps are formed from neutrophils is called Netosis. This process can be initiated by variety of factors, both inorganic and organic. The Netosis inducing agents are listed in Table 1 [5-8]. Neutrophil activation by one of these factors cause initiation of intra- cellular changes within a cell. The cascade of events can vary and it is depended on type of stimuli which activated the cell. Upon activation neutrophils undergo several morphological changes. They start from chromatin decondensation to point when eu- and heterochromatin cannot be distinguished from each other. Furthermore, the characteristic lobular shape of the nucleus is lost. During this time neutrophil elastase (NE) is released from azurophilic granules and it is transported to cell nucleus. Inside this organelle it attaches to $\mathrm{H} 1$ histone protein and degrades it. In the next step of Netosis granule and nuclei membrane diminish. This allows the granular cargo, including myeloperoxidase (MPO) to mix with decondensed chromatin. In the last step, cell membrane ruptures and fully functional trap is released into extracellular matrix (Fig. 1) [9, 10]. Furthermore, Köckritz-Blickwede et al. have showed that eosinophils can survive Etosis process. In their case, the backbone of trap is not constituted from nuclear chromatin but rather from mitochondrial one. In this uncommon event, trap consisted form mt-DNA is catapulted outside the cell preventing its death $[11,12]$. The main function of Extracellular traps is capturing, immobilisation and destruction of pathogens. The active function of extracellular traps is broadly described in modern literature.

Extracellular traps are undeniable simple structures, decorated with proteins derived from the cell, i.e. enzymes,

A
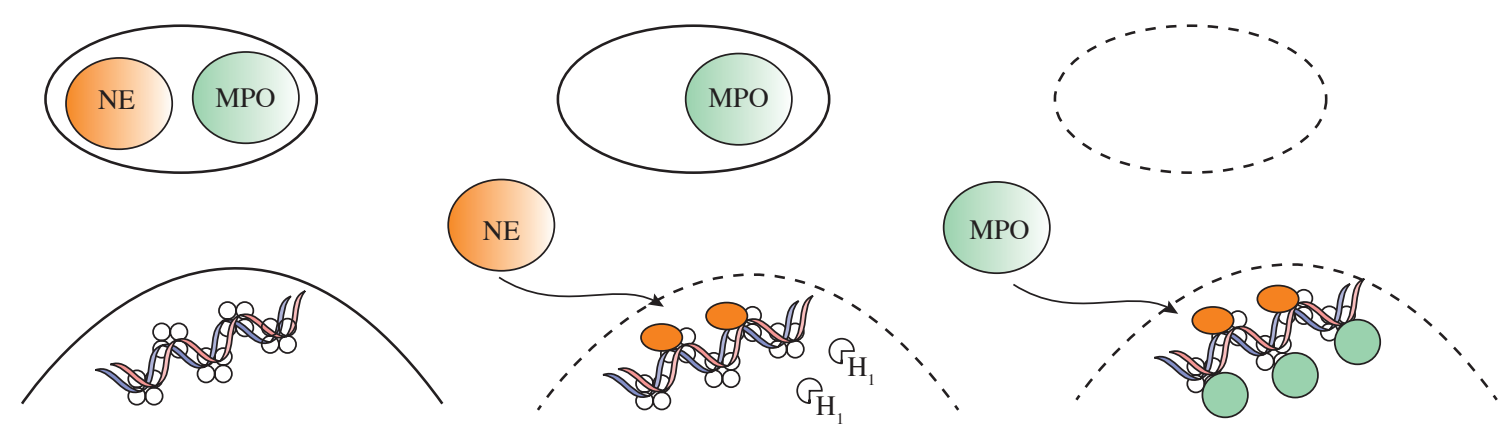

B
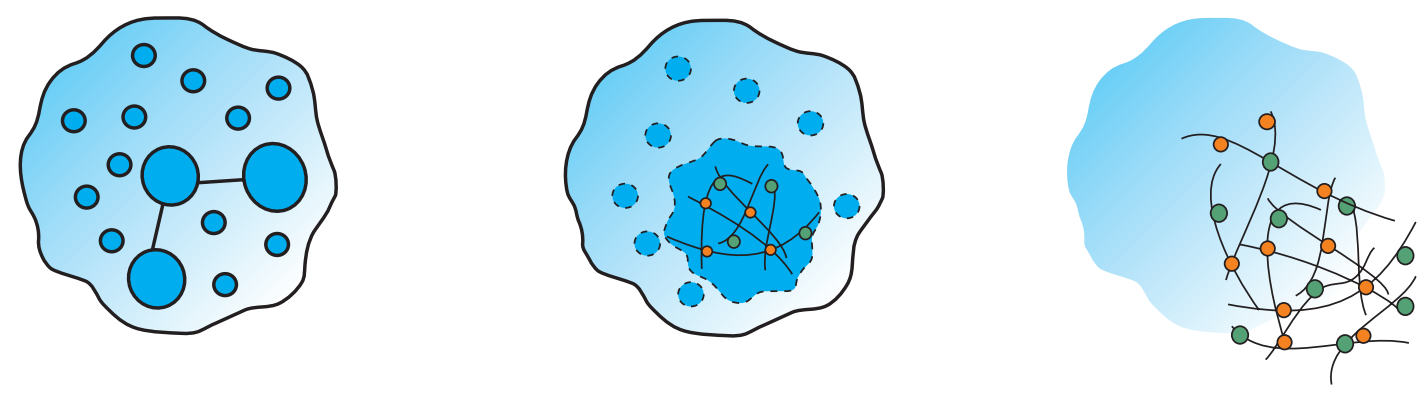

Fig. 1. Neutrophil extracellular traps formation. A) Intracellular events. After decodensation of nuclear chromatin, Neutrophil elastaze is transferred form primary granules to nucleus where it is attached to histone complexes. In result degraded histone $\mathrm{H} 1$ is removed from complex. In the next step, both granular and nuclear membrane diminish and additional proteins like MPO are being incorporated within chromatin strains. B) morphological changes. After formation of vital chromatin trap cell membrane raptures and mature neutrophil trap is released into extracellular matrix 
histones, proteasomes, they act as a circulating web which catch all threats within its DNA based frame and nullifies them, but they can have an impact during autoimmunity.

\section{Nets in autoimmune diseases}

Autoimmune disease is a condition characterised by an abnormal response of the immune system against own structures of the body. During Netosis histones and antigens, that are normally hidden inside neutrophils are released into extracellular matrix. Those antigens are being presented to immunocompetent cells which promote augmented synthesis of autoantibodies. Therefore, it has been postulated that Nets formation may play a key role in pathogenesis of autoimmune disorders [13]. Defects in Nets releasing and clearance and subsequent imbalance between those processes are thought to contribute to the initiation or progression of several autoimmune disorders. The possible connection between Netosis and autoimmunity is supported by the observations, that Plasmodium falciparum infected children show simultaneously increased levels of antinuclear antibodies (ANA) in blood and presence of circulating neutrophil extracellular traps [14]. Other studies also suggest that Netosis may be associated with autoimmune response. Histones are poorly immunogenic proteins, but it has been indicated, that peroxynittrite-modified $\mathrm{H} 2 \mathrm{~A}$ histone became strong immunogen compared with native $\mathrm{H} 2 \mathrm{~A}$ and induced high titre antibodies in rabbits after injection. Peroxynitrite is highly reactive and may cause oxidation and nitration of biomolecules. Since reactive oxygen species can participate in Netosis, it is reasonable to postulate that histones released in this process may be oxidized, and thus, more prone to trigger autoimmune response that native, non-oxidized forms [15]. Further support for this concept bring the fact that MPO or double stranded DNA (dsDNA) that are abundant within extracellular traps, are also structures, against which autoantibodies can be found in patients with autoimmune diseases such as systemic lupus erythematosus (SLE) [13]. According to above findings, it does not seem surprising that a lot of studies explore this ground.

\section{ANCA-associated small-vessel vasculitis}

According to the definition, small-vessel vasculitis (SVV) typically affects arterioles, venules and capillaries, but may also involve arteries and veins. Among SVV there are three diseases, that are associated with the presence of autoantibodies in plasma: Wegener's granulomatosis, Churg-Strauss syndrome and microscopic polyangiitis (MPA) [16]. Both the first and the second condition are necrotizing granulomatous inflammation, and ChurgStrauss is differentiated from Wegener's by the clinical manifestation of asthma and eosinophilia. However these three diseases may show similar symptoms: purpura, pe- ripheral neuropathy, abdominal pain, nephritis and others. They are also associated with the presence of anti-neutrophil cytoplasmic antibodies (ANCA). Target antigens for ANCAs are found in cytoplasm of leukocytes; primary granules of neutrophils and the lysosomes of monocytes contain two major target proteins: myeloperoxidase (MPO) and proteinase 3 (PR3). There are also other polypeptides against whom ANCAs may be produced, such as lactoferrin, elastase or cathepsin G. In laboratory practice ANCAs may be detected with immunofluorescence microscopy and according to staining patterns inside the affected cells there are two primary classes of ANCAs: perinuclear ANCAs (pANCA) for which the most frequent target is MPO and cytoplasmic ANCAs (cANCAs) for which the most frequent antigen is PR3. Most of the patients with SVV have ANCAs in their sera, these autoantibodies seem to be involved in pathogenesis of vasculitis [17]. However, the lack of these autoantibodies does not exclude SVV. Wegener's granulomatosis is usually associated with the presence of PR3-ANCAs (cANCAs), while sera from both MPA and Churg Strauss syndrome patients typically contain anti-MPO antibodies (pANCAs) [18].

As MPO and PR3 are amongst major components of Nets there is possible to be a connection between ANCA vasculitis and Netosis. Recent studies support such a thesis. Kessenbrock et al. examined, whether neutrophils activated by ANCAs are able to form Nets. They reported that IgG isolated from SVV patients sera induced enhanced formation of Nets in neutrophils in vitro compared with control IgG-treated neutrophils. They also showed that these antibodies targeted MPO and PR3 accessible on Nets. The study revealed MPO-DNA complexes present in blood of patients with SVV, especially in those with active disease, that at least partially derived from neutrophils undergoing Netosis. It confirmed previous findings that there are increased levels of nucleosomes in blood of patients with ANCA-vasulitis [19]. Moreover, Kessenbrock et al. found depositions of Nets while analysing inflamed kidney needle biopsies from patients with SVV. In these samples histones, DNA and neutrophil granular proteins were seen in the glomeruli and in the interstitium of the kidneys. They hypothesized, that Netosis is induced mainly during active disease. The authors also proposed the possible role of S. aureus in enhancing Netosis. Staphylococcus aureus is an inducer of Netosis and seems to be associated with relapses during small-vessel vasculitis. In line with above studies, both p-ANCAs and Nets were later found in the epidermis of a woman with cutaneous small-vessel vasculitis [20].

The latest reports have shown, that IgG eluted from MPA patients sera are highly efficient in inducing Netosis. This interaction was especially apparent in samples from patients with high disease activity. Moreover, patients with MPA had significantly lower activity of DNAse I in blood samples than healthy controls and thus, lower rates of Nets 
degradation. These data indicate, that patients with microscopic polyangiitis may show enhanced traps formation simultaneously with disrupted degradation of Nets [21].

It is well-known that patients with small-vessel vasculitis are prone to venous thromboembolic events [22]. As mentioned above, components of Nets might be responsible for binding platelets and blood coagulants, thus contribute to thrombus formation. It has been demonstrated recently that neutrophils from patients with SVV release Nets and microparticles containing tissue factor (TF), a blood clotting agent. Moreover, blood samples collected from patients with active disease demonstrated increased amounts of neutrophil derived microparticles with TF and circulating DNA. Both sera from patients with active disease and IgG isolated from these patients were able to induce the release of TF expressing Nets and microparticles from control neutrophils [16]. There are also other facts indicating that formation of Nets may be associated with an increased risk of deep vein thrombosis in SVV patients. Autopsy materials collected from a patient diagnosed with MPA and complicated with deep vein thrombosis (DVT) revealed some interesting findings. Autopsy showed alveolar hemorrhage with neutrophil infiltration and severe crescentic glomerulonephritis. Nets were abundant not only in the kidneys, which confirmed previous findings in kidneys affected by SVV, but surprisingly they were also present in the thrombus [21]. The amount of Nets in thrombus from MPA patient was significantly greater than in thrombi unrelated to MPA. Histone citrullination plays an important role in Nets formation, accordingly the same study compared the degree of histone citrullination in the thrombus of MPA patient to other thrombi, that were not associated with such a disease. It turned out that the area of citrullination was larger in the patient who suffered from MPA. Apparently Netosis is associated with DVT in patients with small-vessel vasculitis and because of that researchers suggested considering Nets formation as a potential therapeutic target in ANCA-associated vacuities. They proposed that inhibitors of essential for Netosis enzymes, i.e. NADPH oxidase and peptidylarginine deiminase 4 (PAD4), could improve therapeutic outcomes in those patients [23].

An inspiration for further studies became the fact, that MPO ANCAs are found in approximately $30 \%$ of patients treated with antithyroid drug propylothiouracyl (PTU), moreover some of these patients develop ANCA-associated vasculitis [24]. It is also known, that not all the PTU is metabolised in the liver; MPO in neutrophils modifies a part of PTU and then the PTU-metabolites may associate with proteins and trigger cytotoxicity. Furthermore, conformation of heme-proteins was altered after modification of PTU with MPO, thus it is suspected that MPO modification may be the cause of immunization and production of autoantibodies against this enzyme [25]. Nakazawa et al demonstrated that subsequent administration of PTU and
PMA impaired both Nets formation and degradation, not only in vitro, but also in vivo [21]. Their studies support the view, that Nets may be implicated in the pathogenesis of ANCA-vasculitis. An abnormal formation of Nets was expressed by widely extended chromatin fibers after treating neutrophils with PMA, contrary to not extending outward webs after treatment with combination of PMA and PTU. These abnormally Nets were difficult to release into the liquid phase, what also distinguished them from PMA-induced Nets. On the other hand, due to established in the study decreased susceptibility for being degraded by DNase I, abnormal Nets may remain in tissues for too long. It has been hypothesized that PTU metabolites may link to DNA and bother the recognition of cleavage sites by DNase I. Nakazawa et al. also indicated, that both administration of PTU with PMA and abnormal Nets themselves may trigger production of MPO ANCAs and induce clinical signs related with small vessel vasculitis. SVV is connected with onset of crescentic glomerulonephritis and pulmonary hemorrhage due to alveolar capillaritis; some of examined rats developed pulmonary hemorrhage, infiltration of neutrophils around the capillaries and glomerulonephritis $[21,26]$. Above findings support earlier studies on connection between Netosis and SVV and highlight the importance of balance between Nets formation and clearance.

Interestingly, myeloid dendritic cells (mDCs) have been shown recently to capture Net components, such as MPO and PR3, then process and present them as antigens. Moreover, when transferred into a naive mouse, they induced ANCAs and lead to development of subsequent vasculitis. Renal and pulmonary parenchyma were found to show the autoimmune features of vasculitis. Microscopic analysis of skin samples collected from MPA patients also revealed mDCs in close contact with Nets. What is worth noting, the interaction between Netting PMNs and mDCs was possible only when Nets structure was intact, as treatment with DNase I prevented transferring neutrophils autoantigens to $\mathrm{mDCs}$ [27].

After looking complexly at these data we see that $\mathrm{Ne}$ tosis is a key player in autoimmune small vessel vasculitis pathogenesis since Nets are well-known source of MPO and PR3, against which majority of ANCAs are targeted. It is possible that there exist a vicious cycle involving both regulation of Netosis and ANCAs.

\section{Systemic lupus erythematosus}

Systemic lupus erythematosus (SLE) is a severe autoimmune condition, in which the breakdown of self-tolerance leads to the production of autoantibodies. These antibodies are targeted not only against nuclear antigens: histones, DNA, ribonucleoproteins (RNP), but also against granular proteins of neutrophils (ANCA). The disease affects multiple tissues and organs, including kidneys, lungs 
and pleural cavity, skin and heart. Similarly to most of the autoimmune diseases, SLE occurs mainly in women. Its pathophysiology remains not fully clarified yet, but roles of genetics, hormones, environment, lymphocytes abnormalities and other factors have been emphasized [28]. It even was suggested, that ineffective clearance of necrotic and apoptotic cells provides molecules, against which antibodies are synthesized [29]. After Netosis had been described, Nets became considered as a novel source of autoantigens. Extracellular traps contain both chromatin and proteins derived from PMNs granules, so Netosis is likely to be involved in the SLE etiology as well.

It has been established, that a connection between serum endonuclease - DNase I and SLE exists. DNase I-deficient mice were shown to develop similar symptoms to those observed in SLE patients [30]. Moreover, DNase inhibitory antibodies were found in sera from patients with lupus [31], while other studies showed, that there are antibodies binding to DNA which may prevent it from being degraded by DNase [32]. In addition, an occurrence of DNase I gene polymorphism and mutation has been reported in Japanese patients with SLE [33, 34]. Hakkim et al. indicated, that the activity of DNase I was relevant for Nets clearance and this observation led them to examine, whether insufficient Nets degradation by DNase I contributes to SLE. When analysing sera from SLE patients, they found that substantial fraction of them had diminished ability to Net degradation. Scientists termed the subpopulation of patients with poor Net degradation 'non-degraders' and those with higher degradation activity were called 'degraders'. They also discovered, that at least two mechanisms were responsible for impaired Net degradation: the presence of DNase I- specific inhibitors and antibodies shielding Nets from this endonuclease. Also elevated levels of anti-Net antibodies were found in sera from non-degraders [35]. Those results supported previous reports on the role of factors inhibiting DNase I in SLE patients [31, 32]. Furthermore, studies performed by Hakkim et al. showed, that impaired Nets degradation was correlated with higher titers of anti-dsDNA and ANA autoantibodies (which are hallmarks in SLE) and more frequent development of lupus nephritis. They detected Nets with specific IgG in kidney biopsies from non-degrading SLE patients [35]. Further studies corroborated the presence of Netting neutrophils not only in kidneys, but also in skin biopsies from SLE patients with skin involvement $[27,36]$. In line with studies performed by Hakkim also Leffler et al. confirmed that sera from some SLE patients fail to degrade Nets and that sera from non-degraders contained higher levels of antibodies targeted dsDNA and Nets compared to sera from degraders. They also showed, that Net-degrading ability was correlated with disease activity. Higher ability to degrade Nets was observed during remission, while lower was connected with a flare. Moreover, patients in flare had also elevated levels of antibodies against Nets. In line with these results, milder forms of SLE (arthritis and rash) were rarely seen in patients, whose sera were low-degrading. The authors also indicated, that non-degraded Nets could trigger complement activation. They observed an inhibitory effect of C1q on Nets degradation, possibly due to its opsonisation on Nets and direct inhibition of DNase I enzyme [37]. However, these data need to be critically analysed, as recent findings has indicated that $\mathrm{C} 1 \mathrm{q}$ is involved rather in Nets clearance. In addition extremely rare $\mathrm{C} 1 \mathrm{q}$ deficiency results in high risk for developing lupus what suggests protective role of C1q in SLE pathogenesis [38].

Taken together the studies performed by Hakkim and Lefflerg groups, it seems credible that SLE is connected with impaired Net degradation. Mechanisms which are involved in disruption of Nets clearance may become new therapeutic targets. Notably, especially non-degrading patients need special care, as they are more prone to glomerulonephritis. Alternative nucleases or immunoadsorbant columns to remove Net-protecting antibodies could be effective in those patients [35].

There are some evidences, that type I interferons (IFNs) have an important role in the pathogenesis of SLE. Patients with SLE have augmented levels of IFN in sera and present overexpression of interferon-induced genes. Moreover, these parameters are correlated with disease activity $[39,40]$. It has been also described, that complexes containing nucleic acids activate plasmocytoid dendritic cells (pDCs) to produce large amounts of IFN- $\alpha$ in response to Toll-like receptors activation (TLR 9 for DNA and TLR 7 for RNA) [41]. Studies performed by Lande et al suggest, that there is a positive feedback loop involving Netosis and production of IFN- $\alpha$. Immune complexes present in SLE contained components that were released by Netting PMNs were shown to activate pDCs in an TLR9 dependent manner, which in turn released IFN- $\alpha$. LL37 (cathelicidin) and human neutrophil proteins (HNPs) worked synergistically and were necessary to pDCs activation and protection of DNA-containing complexes against degradation by nucleases. The researchers also found anti-LL37 and anti-HNPs antibodies in sera from SLE patients, which activated neutrophils to release Nets. Interestingly, IFN- $\alpha$ enhanced expression of HNP and LL37 on the surface of neutrophils, leading to intensified Nets formation and further pDCs activation. Moreover, it was confirmed that PMNs from SLE patients are more prone to release Nets compared to those from healthy people [42]. Latter studies indicated, that pDCs activation with subsequent IFN- $\alpha$ production may be also triggered by anti-ribonucleoprotein antibodies, able to promote Netosis [43]. Above findings demonstrated, that defective PMNs-pDCS interaction in SLE may lead to self-amplifying feedback loop and contribute a great deal to the disease development and progression. Notably, infections might play an important role in this loop as exogenous agents such as bacteria/ fungi could prime PMNs for additional Netosis. 
Another feed-forward loop relevant in SLE pathogenesis may involve inflammasomes, which are intracellular complexes mediating the immune response via the activation of caspase-1 [44]. LL37 present in Nets and Nets themselves trigger the inflammasome machinery by activation of caspase-1 in macrophages. In turn, activated macrophages release inflammatory cytokines, of which IL-18 may further stimulate Netosis [45]. Moreover, IL-18 contributes to endothelial cells damage and promotes skin and renal inflammation in SLE patients [46].

Interestingly, an abnormal subset of PMNs have been identified in SLE patients as co-purified with the peripheral blood mononuclear cell (PBMC) fractions. These socalled low density granulocytes (LDGs) are proinflammatory cells, they release high levels of type I IFNs (which, as mentioned above, correlates with a disease activity in SLE [30]) and induce endothelial cells cytotoxicity, leading to vascular damage [47]. It has been indicated, that LDGs demonstrate increased ability to form Nets, thus they externalize significant amount of autoantigens such as dsDNA and LL37 to exacerbate the disease progression. It suggests, that not only defective clearance, but also enhanced formation of Nets may be relevant in SLE pathogenesis [36].

As some patients with SLE develop ANCAs, a murine model with lupus prone $1 \mathrm{pr} / \mathrm{lpr}$ mice has been created to examine the possible relationship of this phenomenon with Netosis. The correlation between ANCAs presence in blood samples collected from these mice and increased spontaneous Nets formation by naive PMNs has been shown. Results of the study indicated, that release of extracellular traps in SLE may be the source of both nuclear and cytoplasmic antigens [27].

Unquestionably, a growing body of evidence indicates, that Netosis can contribute to maintenance and progression of SLE. Existence of positive feedback loops suggests that once triggered, Netosis may perpetuate inflammation and promote disease development. Possible new treatment strategies based on modulation of Nets formation and degradation should be the focus of further studies.

\section{Rheumatoid arthritis}

Rheumatoid arthritis (RA) is a long-termed, autoimmune disease, that involves mainly inflammation of joints, but may also affect internal organs. Similarly to SLE, rheumatoid arthritis is more common among women and the elderly. The risk for development of RA is highly dependent on hereditary factors, as $50 \%$ of it is caused by genetic mutations. Overproduction of proinflammatory mediators, such as tumor necrosis factor (TNF) or interleukin 6, leads to perpetuation and exacerbation of this disorder. Moreover, in sera collected from patients with RA a broad range of autoantibodies can be found. These antibodies are anti-filaggrin autoantibodies, anti-keratin antibodies, anti-perinuclear factor, and two most often used for di- agnosis: rheumatoid factor and anticitrullinated peptide antibodies (ACPAs), amongst which particularly relevant are anticyclic citrullinated peptide (ACCs) antibodies. Citrullination (deimination) is the enzymatic conversion of arginine to citrulline in polypeptides and this process may alter antigenicity of proteins. Antibodies directed against citrullinated peptides often appear in sera in the early phases of RA or even before any clinical symptoms develop and they are the serologic hallmark of the disease [48, 49]. Citrullination, as mentioned above, seems to be involved in traps formation, therefore the question arised whether RA and Nets are associated.

Indeed, Khandpur et al. indicated, that there is a correlation between not only Netosis and ACPAs presence and concentration, but also between Nets and systemic inflammatory markers. Moreover, both synovial fluid and circulating neutrophils were more prone to Nets formation than those from healthy controls. They also found deposits of Netting neutrophils in RA synovial tissues, nodules and skin. The same scientists examined if addition of sera or immunoglobulin fractions from RA patients with high levels of ACPAs and/or rheumatoid factor could alter Netosis. They confirmed enhanced Nets formation under these conditions. Further investigations revealed, that Nets induced by autoantibodies contained MMP-8 (matrix-metalloprotease-8), which plays an important role in tissue destruction in RA [14, 50].

Khandpur et al. also showed, that not only autoantibodies from RA sera were capable of inducing Netosis. They found that IL-17a and TNF- $\alpha$ (levels of which are elevated in RA patients sera [51]) can also induce Nets formation in RA neutrophils. Interestingly, these extracellular traps then stimulated synovial fibroblasts to produce IL-6, IL-8, chemokines and adhesion molecules.

Considering these findings together, they hypothesized that Netting neutrophils may externalize citrullinated autoantigens and promote the release of immunostimulatory molecules, which in turn leads to aberrations in immune response and contributes to exacerbation of the disease.

Further support for the role of Nets in RA comes from studies performed by Pratesi et al. They showed that sera obtained from RA patients reacted with proteins isolated from Nets and that the target protein was deiminated histone H4. It suggested that Netosis externalize antigens against which autoantibodies are directed, but we still do not know if it can trigger autoimmunity in a normal or if it is just another puzzle that contributes to the more complex process [52].

\section{Summary}

The autoimmunity research field is at the scope of scientist for many years. During those years new discoveries were made that not only gave answers and expanded current knowledge but they also set up a bunch of new 
mysteries. The latest break in understanding pathophysiology of autoimmune disorders is related with discovery of extracellular traps, but existence of Nets do not answer all question. Thus, in order to extend our lore and find connections between biological pathways we need to go back to past data and try to correspond them with novel findings.

The authors declare no conflict of interest.

\section{References}

1. Yipp BG, Kubes P (2013): NETosis: how vital is it? Blood 122: 2784-2794.

2. Pruchniak MP, Arazna M, Demkow U (2013): Life of neutrophil: from stem cell to neutrophil extracellular trap. Respir Physiol Neurobiol 187: 68-73.

3. Takei H, Araki A, Watanabe H, et al. (1996): Rapid killing of human neutrophils by the potent activator phorbol 12-myristate 13-acetate (PMA) accompanied by changes different from typical apoptosis or necrosis. J Leukoc Biol 59: 229-240.

4. Tsan MF (1980): Phorbol myristate acetate induced neutrophil autotoxicity. J Cell Physiol 105: 327-334.

5. Brinkmann V, Reichard U, Goosmann C, et al. (2004): Neutrophil extracellular traps kill bacteria. Science 303: 1532-5.

6. Branzk N, Papayannopoulos V (2013): Molecular mechanisms regulating NETosis in infection and disease. Semin Immunopathol 35: 513-530.

7. Cheng OZ, Palaniyar N (2013): NET balancing: a problem in inflammatory lung diseases. Front Immunol 4: 1.

8. Guimaraes-Costa AB, Nascimento MT, Froment GS, et al. (2009): Leishmania amazonensis promastigotes induce and are killed by neutrophil extracellular traps. Proc Natl Acad Sci U S A 106: 6748-6753.

9. Fuchs TA, Abed U, Goosmann C, et al. (2007): Novel cell death program leads to neutrophil extracellular traps. J Cell Biol 176: 231-241.

10. Papayannopoulos V, Metzler KD, Hakkim A, et al. (2010): Neutrophil elastase and myeloperoxidase regulate the formation of neutrophil extracellular traps. J Cell Biol 191: 677-691.

11. von Kockritz-Blickwede M, Nizet V (2009): Innate immunity turned inside-out: antimicrobial defense by phagocyte extracellular traps. J Mol Med (Berl) 87: 775-783.

12. Yousefi S, Mihalache C, Kozlowski E, et al. (2009): Viable neutrophils release mitochondrial DNA to form neutrophil extracellular traps. Cell Death Differ 16: 1438-1444.

13. Fattal I, Shental N, Mevorach D, et al. (2010): An antibody profile of systemic lupus erythematosus detected by antigen microarray. Immunology 130: 337-343.

14. Khandpur R, Carmona-Rivera C, Vivekanandan-Giri A, et al. (2013): NETs are a source of citrullinated autoantigens and stimulate inflammatory responses in rheumatoid arthritis. Sci Transl Med 5: 178ra40.

15. Khan MA, Dixit K, Jabeen S, et al. (2009): Impact of peroxynitrite modification on structure and immunogenicity of H2A histone. Scand J Immunol 69: 99-109.

16. Kambas K, Chrysanthopoulou A, Vassilopoulos D, et al. (2014): Tissue factor expression in neutrophil extracellular traps and neutrophil derived microparticles in antineutrophil cytoplasmic antibody associated vasculitis may promote thromboinflammation and the thrombophilic state associated with the disease. Ann Rheum Dis 73: 1854-1863.

17. Kida I, Kobayashi S, Takeuchi K, et al. (2011): Antineutrophil cytoplasmic antibodies against myeloperoxidase, proteinase 3 , elastase, cathepsin $\mathrm{G}$ and lactoferrin in Japanese patients with rheumatoid arthritis. Mod Rheumatol 21: 43-50.

18. Jennette JC, Falk RJ (1997): Small-vessel vasculitis. N Engl J Med 337: 1512-1523.

19. Holdenrieder S, Eichhorn P, Beuers U, et al. (2006): Nucleosomal DNA fragments in autoimmune diseases. Ann N Y Acad Sci 1075: 318-327.

20. Abreu-Velez AM, Smith JG, Jr., Howard MS (2009): Presence of neutrophil extracellular traps and antineutrophil cytoplasmic antibodies associated with vasculitides. N Am J Med Sci 1: 309-313.

21. Nakazawa D, Tomaru U, Yamamoto C, et al. (2012): Abundant neutrophil extracellular traps in thrombus of patient with microscopic polyangiitis. Front Immunol 3: 333.

22. Stassen PM, Derks RP, Kallenberg CG, et al. (2008): Venous thromboembolism in ANCA-associated vasculitis-incidence and risk factors. Rheumatology (Oxford) 47: 530-534.

23. Diamantopoulos AP (2013): Extracellular neutrophil traps: a novel therapeutic target in ANCA-associated vasculitis? Front Immunol 4: 24.

24. Wada N, Mukai M, Kohno M, et al. (2002): Prevalence of serum anti-myeloperoxidase antineutrophil cytoplasmic antibodies (MPO-ANCA) in patients with Graves' disease treated with propylthiouracil and thiamazole. Endocr J 49: 329-334.

25. Lee E, Hirouchi M, Hosokawa M, et al. (1988): Inactivation of peroxidases of rat bone marrow by repeated administration of propylthiouracil is accompanied by a change in the heme structure. Biochem Pharmacol 37: 2151-2153.

26. Nakazawa D, Tomaru U, Ishizu A (2013): Possible implication of disordered neutrophil extracellular traps in the pathogenesis of MPO-ANCA-associated vasculitis. Clin Exp Nephrol 17: 631-633.

27. Sangaletti S, Tripodo C, Chiodoni C, et al. (2012): Neutrophil extracellular traps mediate transfer of cytoplasmic neutrophil antigens to myeloid dendritic cells toward ANCA induction and associated autoimmunity. Blood 120: 3007-3018.

28. Crispin JC, Liossis SN, Kis-Toth K, et al. (2010): Pathogenesis of human systemic lupus erythematosus: recent advances. Trends Mol Med 16: 47-57.

29. Herrmann M, Zoller OM, Hagenhofer M, et al. (1996): What triggers anti-dsDNA antibodies? Mol Biol Rep 23: 265-267.

30. Napirei M, Karsunky H, Zevnik B, et al. (2000): Features of systemic lupus erythematosus in Dnase1-deficient mice. Nat Genet 25: 177-181.

31. Yeh TM, Chang HC, Liang CC, et al. (2003): Deoxyribonuclease-inhibitory antibodies in systemic lupus erythematosus. J Biomed Sci 10: 544-551.

32. Emlen W, Ansari R, Burdick G (1984): DNA-anti-DNA immune complexes. Antibody protection of a discrete DNA fragment from DNase digestion in vitro. J Clin Invest 74: 185-190.

33. Yasutomo K, Horiuchi T, Kagami S, et al. (2001): Mutation of DNASE1 in people with systemic lupus erythematosus. Nat Genet 28: 313-314.

34. Shin HD, Park BL, Kim LH, et al. (2004): Common DNase I polymorphism associated with autoantibody production among systemic lupus erythematosus patients. Hum Mol Genet 13: 2343-2350. 
35. Hakkim A, Furnrohr BG, Amann K, et al. (2010): Impairment of neutrophil extracellular trap degradation is associated with lupus nephritis. Proc Natl Acad Sci U S A 107: 9813-9818.

36. Villanueva E, Yalavarthi S, Berthier CC, et al. (2011): Netting neutrophils induce endothelial damage, infiltrate tissues, and expose immunostimulatory molecules in systemic lupus erythematosus. J Immunol 187: 538-552.

37. Leffler J, Martin M, Gullstrand B, et al. (2012): Neutrophil extracellular traps that are not degraded in systemic lupus erythematosus activate complement exacerbating the disease. J Immunol 188: 3522-3531.

38. Troedson C, Wong M, Dalby-Payne J, et al. (2013): Systemic lupus erythematosus due to $\mathrm{C} 1 \mathrm{q}$ deficiency with progressive encephalopathy, intracranial calcification and acquired moyamoya cerebral vasculopathy. Lupus 22: 639-643.

39. Ytterberg SR, Schnitzer TJ (1982): Serum interferon levels in patients with systemic lupus erythematosus. Arthritis Rheum 25: 401-406.

40. Bennett L, Palucka AK, Arce E, et al. (2003): Interferon and granulopoiesis signatures in systemic lupus erythematosus blood. J Exp Med 197: 711-723.

41. Barrat FJ, Meeker T, Gregorio J, et al. (2005): Nucleic acids of mammalian origin can act as endogenous ligands for Tolllike receptors and may promote systemic lupus erythematosus. J Exp Med 202: 1131-1139.

42. Lande R, Ganguly D, Facchinetti V, et al. (2011): Neutrophils activate plasmacytoid dendritic cells by releasing selfDNA-peptide complexes in systemic lupus erythematosus. Sci Transl Med 3: 73ra19.

43. Garcia-Romo GS, Caielli S, Vega B, et al. (2011): Netting neutrophils are major inducers of type I IFN production in pediatric systemic lupus erythematosus. Sci Transl Med 3: $73 \mathrm{ra} 20$.

44. Lamkanfi M, Dixit VM (2012): Inflammasomes and their roles in health and disease. Annu Rev Cell Dev Biol 28: 137161.

45. Kahlenberg JM, Carmona-Rivera C, Smith CK, et al. (2013): Neutrophil extracellular trap-associated protein activation of the NLRP3 inflammasome is enhanced in lupus macrophages. J Immunol 190: 1217-1226.

46. Wang D, Drenker M, Eiz-Vesper B, et al. (2008): Evidence for a pathogenetic role of interleukin-18 in cutaneous lupus erythematosus. Arthritis Rheum 58: 3205-3215.

47. Denny MF, Yalavarthi S, Zhao W, et al. (2010): A distinct subset of proinflammatory neutrophils isolated from patients with systemic lupus erythematosus induces vascular damage and synthesizes type I IFNs. J Immunol 184: 3284-3297.

48. Chang X, Yamada R, Suzuki A, et al. (2005): Localization of peptidylarginine deiminase 4 (PADI4) and citrullinated protein in synovial tissue of rheumatoid arthritis. Rheumatology (Oxford) 44: 40-50.

49. Kuhn KA, Kulik L, Tomooka B, et al. (2006): Antibodies against citrullinated proteins enhance tissue injury in experimental autoimmune arthritis. J Clin Invest 116: 961-973.

50. Malemud CJ (2006): Matrix metalloproteinases (MMPs) in health and disease: an overview. Front Biosci 11: 1696-701.

51. Kim J, Kang S, Kwon G, et al. (2013): Elevated levels of T helper 17 cells are associated with disease activity in patients with rheumatoid arthritis. Ann Lab Med 33: 52-59.

52. Pratesi F, Dioni I, Tommasi C, et al. (2013): Antibodies from patients with rheumatoid arthritis target citrullinated histone 4 contained in neutrophils extracellular traps. Ann Rheum Dis 73: $1414-1422$. 